\title{
INCERTIDUMBRE FRENTE A LA ENFERMEDAD EN MUJERES DIAGNOSTICADAS CON CÁNCER DE SENO
}

Dennys Isabel Muñoz Monterroza ${ }^{1}$, Regina Cogollo Jiménez ${ }^{2}$, Aníbal Arteaga Noriega ${ }^{3}$, Sandra Hernández Pérez ${ }^{4}$

\section{Resumen}

El cáncer de seno (o cáncer de mama) es una enfermedad crónica que puede generar incertidumbre, afectar la salud integral y comprometer la calidad de vida de la paciente. Estudio correlacional, cuantitativo y transversal que se hizo con el objetivo de relacionar el grado de incertidumbre de las mujeres con cáncer de seno, según variables sociodemográficas y de diagnóstico. La muestra se realizó con 61 participantes, mediante muestreo por conveniencia. Se utilizó un cuestionario de datos sociodemográficos y la escala de incertidumbre de Mishel. El procesamiento y análisis de datos se realizó a través de SPSS 19 y la relación entre las variables mediante Chi-cuadrado. La mayoría de la población en estudio tiene una edad entre 50 y 69 años. El $62 \%$ presenta regular grado de incertidumbre. El grado de incertidumbre en la población estudiada está relacionado con las variables: nivel educativo y tiempo de diagnóstico.

1 Enfermera. Magíster en Salud Pública y Sistemas de Salud. Docente del Programa de Enfermería, Facultad de Ciencias de la Salud, Universidad del Sinú -Elías Bechara Zainum-Colombia. dennysisabelm83@gmail.com

${ }^{2}$ Enfermera. Magíster en Enfermería. Docente del Departamento de Enfermería, Facultad de Ciencias de la Salud, Universidad de Córdoba - Colombia. recogojim@ hotmail.com

3 Enfermero. Magíster en Salud Pública y Planificación Sanitaria. Docente del Programa de Enfermería, Facultad de Ciencias de la Salud, Corporación Universidad Remington - Colombia. anviarno@gmail.com.

${ }^{4}$ Enfermera egresada de laUniversidad del Sinú-Elías Bechara Zainum - Colombia. 


\section{UNCERTAINTY AGAINST DISEASE IN DIAGNOSED WOMEN WITH BREAST CANCER}

Dennys Isabel Muñoz Monterroza, Regina Cogollo Jiménez, Aníbal Arteaga Noriega, Sandra Hernández Pérez

\section{Abstract}

Breast cancer is a chronic disease that can generate uncertainty, affect health in different ways and compromise the quality of life of the patient. Correlational, quantitative and cross-sectional study that was carried out with the aim of relating the level of uncertainty of women with breast cancer, according to sociodemographic and diagnostic variables. The sample was performed to 61 participants, by means of convenience sampling. A socio-demographic data questionnaire and Mishel's uncertainty test were used. The processing and analysis of data was developed through the SPSS 19 and the relationship between the variables by Chi-square. The majority of the study population is between 50 and 69 years old. $62 \%$ have a regular level of uncertainty. The level of uncertainty in the population studied is related to the variables: the level of education and time of diagnosis. 


\section{INCERTEZA NA DOENÇA EM MULHERES DIAGNOSTICADAS COM CÂNCER DE MAMA}

Dennys Isabel Muñoz Monterroza, Regina Cogollo Jiménez, Aníbal Arteaga Noriega, Sandra Hernández Pérez

\section{Resumo}

O câncer de mama é uma doença crônica que pode gerar incertezas, afetar a saúde geral e comprometer a qualidade de vida do paciente. Estudo correlacional, quantitativo e transversal, realizado com o objetivo de relacionar o grau de incerteza de mulheres com câncer de mama, segundo variáveis sociodemográficas e diagnósticas. A amostra foi composta de 61 participantes, por meio de amostragem por conveniência. Utilizou-se um questionário de dados sociodemográficos e a escala de incerteza de Mishel. O processamento e análise dos dados foram feitos através do SPSS 19 e a relação entre as variáveis foi determinada mediante o Qui-quadrado. A maioria da população do estudo tem entre 50 e 69 anos de idade. $62 \%$ apresenta um grau regular de incerteza. O grau de incerteza na população estudada está relacionado às variáveis: escolaridade e tempo de diagnóstico. 


\section{Introducción}

La epidemiologia del cáncer de seno ha cambiado de manera importante durante los últimos años, por cuanto se observa aumento en la prevalencia, la mortalidad, los costos económicos y en tal sentido, se convierte en un problema de salud pública (1). En los países de ingresos medios a bajos el problema es mayor, debido a las falencias en los sistemas de salud para la detección oportuna de la enfermedad, lo que conlleva a un diagnóstico en estadios avanzados (2).

El cáncer de seno no solo afecta a la persona que sufre la enfermedad (3), sino también se percibe como una experiencia que influye a toda la familia, porque los roles de todos los miembros se ven considerablemente afectados (4), creándose un trastorno en la rutina diaria familiar y en la habitual disponibilidad de la madre, lo que puede conllevar a conflictos y estrés $(5,6)$.

Para el tratamiento del cáncer de seno es importante un abordaje integral, por ser considerada una enfermedad crónica, en la cual la evolución de la misma depende de múltiples factores y muchas veces es indeterminable la sintomatología del paciente, causando un impacto negativo en la persona y en el núcleo que lo rodea $(7,8)$. La incertidumbre puede ser uno de los factores que deterioran la tranquilad y el bienestar integral de la persona afectada, especialmente en el diagnóstico, pues la inseguridad, la angustia y el enfrentarse a la muerte puede generar afectaciones físicas, emocionales y espirituales, y pueden acelerar el desarrollo de la enfermedad o generar la aparición de otras patologías, comprometiendo su calidad de vida $(9,10)$.

Desde la disciplina de enfermería existe una teoría que habla de la incertidumbre frente a la enfermedad, creada por Merle H. Mishel (11), que ha sido utilizada para describir el proceso de adaptación a diversas patologías $(9,12,13)$. Esta teoría describe la incertidumbre como la incapacidad de describir e interpretar el significado de los hechos relacionados con la enfermedad, ocasionando una predicción ineficaz de los resultados $(9,12)$, afectando el afrontamiento del paciente ante la nueva condición.

La incertidumbre está relacionada con desconocimiento y desinformación sobre el proceso de la enfermedad, lo que incluye los procedimientos diagnósticos, el tratamiento y seguimiento. La identificación de la incertidumbre por el personal de salud puede orientar las estrategias de intervención que promuevan la adaptación y el afrontamiento del paciente y la familia ante la experiencia de la enfermedad $(14,15)$. Sin embargo, es importante considerar los elementos conceptuales planteados en el modelo como son el esquema, las capacidades cognitivas y el apoyo social, los cuales pueden estar influidos por las condiciones propias del paciente como lo son las variables sociodemográficas.

De acuerdo con la problemática descrita surge la presente investigación, la cual tiene como objetivo relacionar el grado de incertidumbre de las mujeres con cáncer de seno, según las variables de edad, nivel educativo, estado civil, nivel socioeconómico y tiempo de diagnóstico. 


\section{Materiales y Métodos}

\section{Tipo de estudio}

El tipo de diseño es cuantitativo, correlacional, de corte trasversal, lo cual permite identificar el grado de incertidumbre de las mujeres con cáncer de seno, teniendo en cuenta la teoría de la incertidumbre de Merle H. Mishel (11), y su relación con las variables edad, nivel educativo, estado civil, nivel socioeconómico y tiempo de diagnóstico.

\section{Población, muestra y muestreo}

Mediante un muestreo no probabilístico por conveniencia se seleccionaron 61 mujeres mayores de 18 años, con diagnóstico de cáncer de seno, en una IPS privada de cuarto nivel de atención, en la ciudad de Montería, entre los meses de marzo y julio de 2016.

\section{Instrumentos}

Para el logro de los objetivos se utilizó una prueba piloto previa y un cuestionario sociodemográfico diseñado por los autores, que permitieron realizar los ajustes necesarios. Además de utilizó la escala de incertidumbre creada por Merle $\mathrm{H}$. Mishel con su versión validada en español (14), la cual se ha implementado en diversas investigaciones en Colombia $(9,16)$. La escala presenta cuatro factores: ambigüedad, predictibilidad, complejidad e inconsistencias. Contiene 29 preguntas de tipo Likert de 1 a 5 puntos y va desde muy en desacuerdo a muy acuerdo; un puntaje de 5 de cada pregunta refleja un nivel alto de incertidumbre, excepto en las preguntas $6,7,10,12,21,22,25,27$ y 29 , en las cuales el puntaje es invertido. Se puede obtener un puntaje máximo de 145 puntos y un mínimo de 29 puntos. Un puntaje $<59$ puntos es bajo nivel de incertidumbre (NI); regular corresponde a 59-87 puntos y alto NI: $>87$ puntos (16). La escala ha sido aplicada ampliamente en diferentes poblaciones de pacientes, demostrando ser un instrumento fiable y válido para la medida de la incertidumbre. Se realizó una prueba piloto, estableciendo fácil comprensión de la escala y un tiempo de aplicación promedio de 30 minutos.

\section{Análisis de los datos}

El procesamiento de la información se realizó a través de SPSS 19. Para el análisis de los datos se utilizaron medidas descriptivas y de tendencia central. La relación entre las variables se determinó mediante la prueba de independencia Chi-cuadrado.

\section{Consideraciones éticas}

Según la Resolución 8430 de 1993, la presente investigación es considerada una investigación sin riesgo, porque no se va a intervenir físicamente al paciente, siendo una investigación de tipo documental, por medio de un cuestionario y el instrumento es la escala de incertidumbre, por lo cual no se alteró ni se modificaron las variables obtenidas de la información de la muestra. A cada participante se le solicitó autorización por medio del consentimiento informado previamente firmado. 


\section{Resultados}

Según los aspectos sociodemográficos, el $42 \%$ de las participantes se encontraban en el intervalo de 50-69 años; 31,1\% de las participantes estaban casadas, el $11,5 \%$, separadas, el $21,3 \%$, solteras, el 29, 5\%, en unión libre y el 6,6\% de la población en estudio eran viudas. En cuanto al nivel educativo, el 4,9\% de las participantes no realizó ningún estudio, el 23\% cursó la primaria, el 4,6\% estudió el bachillerato, el 27,9\% se graduó como técnico-universitario y el 1, 63\% realizó estudios de posgrado.

En relación con el nivel socioeconómico, el $85,2 \%$ de las participantes pertenece a los estratos 1 y 2 . Por su parte, teniendo en cuenta el tiempo de diagnóstico, el 57,4\% de las participantes habían sido diagnosticadas desde hace 13 a 24 años. (Tabla 1).

Tabla 1. Variables sociodemográficas y tiempo de diagnóstico de las mujeres con cáncer de seno participantes del estudio.

\begin{tabular}{lcc}
\multicolumn{1}{c|}{ Variables } & Frecuencia & Porcentaje (\%) \\
\hline Edad & & \\
18-30 años & 11 & $18 \%$ \\
31-49 años & 21 & $34,4 \%$ \\
50-69 años & 26 & $42,6 \%$ \\
70 años o más & 3 & $4,9 \%$ \\
\hline Estado civil & & \\
Soltera & 13 & $21,3 \%$ \\
Casada & 19 & $31,1 \%$ \\
Separada & 7 & $11,5 \%$ \\
Unión libre & 18 & $29,5 \%$ \\
Viuda & 4 & $6,6 \%$ \\
\hline Nivel educativo & & \\
Sin estudios & 3 & $4,9 \%$ \\
Primaria & 14 & $23 \%$ \\
Secundaria & 26 & $42,6 \%$ \\
Técnica-universitaria & 17 & $27,9 \%$ \\
Postgrado & 1 & $1,6 \%$ \\
\hline Nivel socioeconómico & & \\
Estrato 1-2 & 52 & $85,2 \%$ \\
Estrato 3-4 & 8 & $13,1 \%$ \\
Estrato 5-6 & 1 & $1,6 \%$ \\
\hline Tiempo de diagnóstico & & \\
Menos de 6 meses & & $8,2 \%$ \\
6-12 meses & & $28 \%$ \\
13-24 meses & 5 & $57,4 \%$ \\
Más de 24 meses & 17 & $6,6 \%$ \\
\hline
\end{tabular}


Respecto al nivel de incertidumbre, el $62 \%$ de las participantes presentó un grado regular de incertidumbre, de acuerdo con la clasificación realizada por Merle. H. Mishel.

Tabla 2. Nivel de incertidumbre frente al cáncer de seno en las mujeres participantes del estudio.

\begin{tabular}{cc|c|c|c|c|}
\hline & Frecuencia & Porcentaje & $\begin{array}{c}\text { Porcentaje } \\
\text { válido }\end{array}$ & $\begin{array}{c}\text { Porcentaje } \\
\text { acumulado }\end{array}$ \\
\hline Válido & bajo & 0 & 0 & 0 & 0 \\
& regular & 38 & 62,3 & 62,3 & 62,3 \\
& alto & 23 & 37,7 & 37,7 & 100,0 \\
& 61 & 100,0 & 100,0 & \\
\hline
\end{tabular}

Fuente: Cuestionario sociodemográfico. Montería, 2016.

El mayor índice de incertidumbre se presentó ante el proceso de la enfermedad (no saben si ha empeorado o avanzado), ante las explicaciones por parte del médico (le parecen confusas, no las entienden), dificultad para entender el tratamiento (la finalidad del tratamiento no es clara), miedo frente al dolor, dificultad para hacer frente a los síntomas. Presentan incertidumbre en el futuro y en su estado de ánimo diario.
Respecto a la relación entre los aspectos sociodemográficos, tiempo de diagnóstico y el nivel de incertidumbre mediante la prueba Chi-cuadrado se obtuvo para las variables nivel educativo $(p=0,021)$ $\mathrm{y}$ tiempo de diagnóstico $(\mathrm{p}=0,006)$ una asociación entre dichas variables y el nivel de incertidumbre. Para el resto de las variables estudiadas no se observó una asociación significativa, siendo el valor p mayor que 0,05 (Tabla 3). 
Tabla 3. Relación de las variables sociodemográficas y tiempo de diagnóstico con el nivel de incertidumbre en las mujeres participantes del estudio.

\begin{tabular}{|c|c|c|c|c|}
\hline \multirow[t]{2}{*}{ Variables } & \multicolumn{2}{|c|}{ Nivel de incertidumbre } & \multirow[t]{2}{*}{$\begin{array}{c}\text { Chi- cuadra- } \\
\text { do } \\
\end{array}$} & \multirow[t]{2}{*}{ Sig. } \\
\hline & Regular & Alto & & \\
\hline \multicolumn{5}{|l|}{ Edad } \\
\hline 18-30 años & 4 & 7 & \multirow{4}{*}{6,48} & \multirow{4}{*}{0,90} \\
\hline 31-49 años & 12 & 9 & & \\
\hline 50-69 años & 19 & 7 & & \\
\hline 70 años o más & 3 & 0 & & \\
\hline \multicolumn{5}{|l|}{ Estado civil } \\
\hline Soltera & 6 & 7 & \multirow{5}{*}{4,0} & \multirow{5}{*}{0,39} \\
\hline Casada & 12 & 7 & & \\
\hline Separada & 7 & 3 & & \\
\hline Unión libre & 12 & 6 & & \\
\hline Viuda & 4 & 0 & & \\
\hline \multicolumn{5}{|l|}{ Nivel educativo } \\
\hline Sin estudios & 3 & 0 & \multirow{5}{*}{11,5} & \multirow{5}{*}{0,021} \\
\hline Primaria & 13 & 1 & & \\
\hline Secundaria & 14 & 12 & & \\
\hline Técnica-universitaria & 8 & 9 & & \\
\hline Postgrado & 0 & 1 & & \\
\hline \multicolumn{5}{|l|}{ Nivel socioeconómico } \\
\hline Estrato 1-2 & 34 & 18 & \multirow{3}{*}{2,3} & \multirow{3}{*}{0,30} \\
\hline Estrato 3-4 & 4 & 4 & & \\
\hline Estrato 5-6 & 0 & 1 & & \\
\hline \multicolumn{5}{|l|}{ Tiempo de diagnóstico } \\
\hline$<$ de 6 meses & 0 & 5 & \multirow{4}{*}{12,5} & \multirow{4}{*}{0,006} \\
\hline De 6-12 meses & 9 & 8 & & \\
\hline De 13-24 meses & 25 & 10 & & \\
\hline$>$ de 24 meses & 4 & 0 & & \\
\hline
\end{tabular}

Fuente: Cuestionario sociodemográfico - Escala de incertidumbre. Montería, 2016.

\section{Discusión de resultados}

El $62 \%$ de las participantes presentó un nivel regular de incertidumbre frente al cáncer de seno. Este resultado concuerda con lo reportado en un estudio realizados en pacientes diabéticos en Cartagena (15). Sin embargo, difiere con un estudio realizado en pacientes oncológi- cos en Chile (17) y otro en pacientes con cardiopatía isquémica, en el cual el nivel de incertidumbre fue alto (16).

Se ha descrito que la apreciación de la incertidumbre constituye un reflejo de la subjetividad en la experiencia de enfrentarse a la muerte o a los cambios que produce una enfermedad y las res- 
puestas de afrontamientos para la adaptación (18). La incertidumbre refleja una serie de sentimientos que influyen directamente sobre las estrategias de afrontamiento que puede tener la persona respecto a una enfermedad, un nivel de incertidumbre regular indica que, a pesar de procesar cierta información, las pacientes siguen teniendo dificultad para lograr la adaptación a su nueva condición de vida (8).

El rango etario que predominó en las participantes del estudio es de 50 a 69 años. Concuerda con lo descrito por Montalvo et al (19) y Mera (2), cuyo promedio de edad observada en sus estudios fue de 54 y 52.8 años respectivamente. Según el Centro para el Control y la Prevención de Enfermedades (CDC), el riesgo de padecer cáncer de seno se incrementa conforme aumenta la edad (20).

En cuanto al estado civil de las participantes se encontró que el $31,1 \%$ son casadas y el $29,1 \%$ se encuentra en unión libre. En un estudio realizado en Antioquia se informó que la mayoría de las mujeres que recibían tratamiento para esta patología tenían una pareja permanente (54\%) y esta condición les favoreció por el apoyo que recibían (21). Se ha descrito que las expresiones de amor y transmisión de confianza ayudan a enfrentar las crisis emocionales presentadas durante el proceso de la enfermedad (22). El apoyo social es un protector de la salud en todas las personas, en este caso, en las mujeres con cáncer de seno y se relaciona con menores niveles de incertidumbre (23) y mejor calidad de vida en pacientes oncológicos (24). Sin embargo, en el análisis estadístico no se observó una asociación significativa entre el estado civil y el nivel de incertidumbre en el presente estudio.

Con relación al nivel educativo de las participantes se encontró que un $42.62 \%$ es bachiller, es decir, tienen una educación media o secundaria, similar a lo encontrado en pacientes con cáncer de mama en España (25). Estudios han descrito al nivel educativo como factor determinante de la salud mental, el bienestar y la salud percibida $(26,27)$. Así mismo, se ha descrito que la educación se asocia con menores niveles de incertidumbre $(9,28,29)$, y presenta un impacto directo en esta, en las personas con mayor nivel educativo, convirtiéndola en un aspecto positivo con mayor prontitud frente a aquellos con un nivel de educación bajo (19). El tener un nivel de educación medio o superior contribuye a mejorar el entendimiento de las situaciones estresantes a las que se enfrenta la persona ante una enfermedad (13).

Respecto al nivel socioeconómico, el $85,2 \%$ de las participantes son de estrato 1 y 2 , es decir, están en un nivel socioeconómico bajo; sin embargo, no se observó asociación con el nivel de incertidumbre $(\mathrm{p}=0,30)$, lo que es contrario a lo descrito en la literatura, donde se describe al nivel socioeconómico como un factor estrechamente relacionado con el estado de salud (30). Diversos estudios revelan que el menor nivel socioeconómico está asociado a peores estados de salud (31) y determina en muchos casos, el acceso oportuno a los servicios de sanitarios, lo que influye en la detección temprana de los diferentes tipos de cáncer (32), entre ellos el de seno y en una mayor eficacia en el manejo de la enfermedad, incidiendo 
En relación con el tiempo de diagnóstico, se observó una relación, es decir entre mayor es el tiempo de diagnóstico, menor es el nivel de incertidumbre. Lo que concuerda con lo descrito en la literatura, donde se ha establecido que, a mayor tiempo de diagnóstico, el paciente y su familia se va familiarizando con la experiencia de la enfermedad, adquiriendo mayor conocimiento y por ende, disminuyendo la ansiedad y la incertidumbre que la nueva condición genera $(3,4,5)$.

\section{Conclusiones}

Participaron en el presente estudio, 61 mujeres. El 62\% presentó regular grado de incertidumbre y el 38\% niveles altos. Como respuesta al objetivo principal se determinó que las variables nivel educativo y tiempo de diagnóstico se asocian con la incertidumbre frente a la enfermedad en mujeres con cáncer de seno. Por su parte, las variables edad, nivel socioeconómico, estado civil no se asociaron a un mayor nivel de incertidumbre en la población estudiada.

\section{Conflicto de interés}

Los autores de esta investigación declararon no tener conflictos de intereses por motivos intelectuales, académicos, morales e investigativos. Este proyecto fue realizado con recursos propios.

4. Díaz Castellanos I, et al. Afrontamiento frente a la enfermedad en pacientes oncológicos. Rev Ciencia y Cuidado. Enero - diciembre, 2010;7(1):61-70. ISSN 17949831. E-ISSN 2322-7028. Disponible en: http://revistas.ufps.edu.co/ojs/index.php/ cienciaycuidado/article/view/494

5. Arving $C$, et al. Individual psychosocial support for breast cancer patients: a randomized study of nurse versus psychologist interventions and standard care. Cancer Nurs. May-june, 2007;30(3):10-9. Disponible en :http://dx.doi.org/10.1097/01. NCC.0000270709.64790.05

6. Rincón ME, Pérez MÁ, Borda M, Martín A. Impacto de la reconstrucción mamaria sobre la autoestima y la imagen corporal en pacientes con cáncer de mama. Universitas Psychologica (Bogotá) Ene-mar 2012;11(1):25-41. EISSN 2011-2777.
20 tegral para supervivientes. Aten Primaria. Mayo 2012;44(5):288-292. Doi: https:// doiorg/10.1016/j.aprim.2011.07.011. 
7. Arreguin L, Morales MC, Bonilla LM, Soriano MM, Tlalpan RMT, Pérez V. Incertidumbre y calidad de vida en mujeres y hombres afectados por cáncer. Enfermería cuidados humanizados (Montevideo) 2012;1(2):77-83. E-ISSN 2396-6606. Disponible en: http://dx.doi.org/10.22235/ ech.v1i2.829

8. Gómez IP, Castillo IY, Alvis LR. Incertidumbre en adultos diabéticos tipo 2 a partir de la teoría de Merle Mishel. Aquichan (Bogotá) 2015;15(2):210-218. Disponible en: http://dx.doi.org/10.5294/ aqui.2015.15.2.5

9. Falo Zamora C, Villar Abelló H, Rodríguez Bruzos E, Mena Cervignon M, Font Guiteras A. Calidad de vida en cáncer de mama metastásico de larga evolución: la opinión de las pacientes. Psicooncología. 2014;11(2-3):313-31

10. Carrillo González GM, Gómez Ramírez OJ, Arias EM. Teorías de enfermería para la investigación y práctica en cuidado paliativo. Revista Latinoamericana de Bioética. 2016;17(1):60-79. Disponible en: http://dx.doi.org/10.18359/rlbi.1764

11. Triviño Martínez Á, Solano Ruiz MC, Siles González J. Aplicación del modelo de incertidumbre a la fibromialgia. Atención primaria. Abril 2016;48(4):219-255. Disponible en: https://doi.org/10.1016/j. aprim.2015.04.007

12. Peñaloza García M, Mora Merchan MA, Jaimes Medina AY, Quintero AI. Percepción de la muerte en personas portadoras del virus de la inmunodeficiencia humana (VIH) en situación de síndrome de inmunodeficiencia adquirida (SIDA). Rev Ciencia y Cuidado. 2013;10(1):1118. ISSN 1794-9831. E-ISSN 2322-7028. Disponible en: http://revistas.ufps.edu. co/ojs/index.php/cienciaycuidado/article/ view/274 Citado: sep. 2016

13. Torres Ortega C, Peña Amaro P. Adaptación cultural de la Escala de Incertidumbre de Mishel en el paciente tratado con hemodiálisis. Enferm Clin. January-fe- bruary 2015;25(1):9-18. Doi https://doi. org/10.1016/j.enfcli.2014.09.002

14. Cerón ML, Argote, LÁ. La inasistencia al Programa Madre Canguro desde la teoría de la incertidumbre y del modelo Dreyfus. Avances en Enfermería. 2012;30:7082. ISSN 0121-4500. E-ISSN 2346-0261. Disponible en: http://dx.doi.org/10.15446/ av.enferm

15. Gómez Palencia IP, Durán de Villalobos MM. Espiritualidad e incertidumbre ante la enfermedad: adultos diabéticos tipo 2. Avances en Enfermería. 2012;30:1828. ISSN 2346-0261. Disponible en: http:// dx.doi.org/10.15446/av.enferm

16. Flórez Torres IE, Ruidiaz Charris RL, González Hernández J. Valoración de la incertidumbre en pacientes con cardiopatía isquémica. Revista Actual. Enferm. Julio -septiembre 2014;17(3):15-21. Disponible en: https://encolombia.com/medicina/revistas-medicas/enfermeria/ve173/comite173/

17. Johnson Castro MI, Hurtado Arenas P. La incertidumbre frente a la enfermedad en adultos mayores con diagnóstico de cáncer. Cienc. enferm. [Internet]. 2017; 23(1):57-65. ISSN 0717-2079. Disponible en: http://dx.doi.org/10.4067/S071795532017000100057.

18. Alonso JP. La incertidumbre como producción colectiva: ambigüedades en torno al pronóstico en enfermedades terminales. Rev. Runa. (Ciudad Autónoma de Buenos Aires) enero-julio 2013;34(1):11332. ISSN 0325-1217. E-ISSN 1851-9628. Disponible en: http://www.scielo.org.ar/ pdf/runa/v34n1/v34n1a08.pdf

19. Montalvo Prieto AA, et al. Condiciones sociodemográficas y nivel de incertidumbre en mujeres ante el diagnóstico de Cáncer de mama. Rev. Hacia la promoción de la salud. 2016;21(2):114-126. ISSN 0121-7577 http://dx.doi.org/10.17151/hpsal.2016.21.2.9.

20. Centro para el Control y la Prevención de Enfermedades. Programa Nacional de 
Control Integral del Cáncer 2015. Disponible en: https://www.cdc.gov/spanish/cancer/breast/statistics/age.htm .

21. Salas Zapata C, Grisales Romero H. Calidad de vida y factores asociados en mujeres con cáncer de mama en Antioquia, Colombia. Rev. Panam Salud Publica. (Washington) Julio 2010;28(1):9-18. Disponible en: http://dx.doi.org/10.1590/ S1020-49892010000700002.

22. Cardoso, MJ, Ramos, MI, Vaz, FJ, Rodríguez, L, Fernández, N. Influencia del apoyo familiar en momentos de gran incertidumbre. Prisma Social [Internet]. 2012;(8):28-47. ISSN 1989-3464. Disponible en: http://www.redalyc.org/articulo. oa? id=353744580002.

23. Hernández Moreno F, Landero Hernández R. Propiedades psicométricas de la escala de felicidad subjetiva (SHS) y su relación con el estrés, la salud percibida y el apoyo social en pacientes con cáncer de mama. Psicooncología. Norteamérica. Noviembre 2014;11(2-3):357-367. ISSN: 1696-7240. Disponible en: http:// dx.doi.org/10.5209/rev_PSIC.2014.v11. n2-3.47394.

24. Coyotl E, et al. Relación del apoyo social y la calidad de vida en personas con cáncer. European Journal of Health Research 2015; 1(2): 51-61. ISSN 24449067. Disponible en: http:// www.ejhr.es doi: 10.1989/ejhr.v1i2.5

25. Abt Sack A, Et al. Necesidades de información y uso de Internet en pacientes con cáncer de mama en España. Gac. Sanit. Barcelona, may-jun. 2013;27(3):241-247. Disponible en: http://dx.doi.org/10.1016/j. gaceta.2012.06.014

26. De la Cruz E, Feu S, Vizuete-Carrizosa M. El nivel educativo como factor asociado al bienestar subjetivo y la salud mental en la población española. Universitas Psychologica. (Bogotá) 2013;12(1):31-40.

22 ISSN 1657-9267. Disponible en: http://revistas.javeriana.edu.co/index.php/revPsycho/article/viewFile/1735/3960
27. Aguilar Palacio I, Carrera Lasfuentes P, Rabanaque MJ. Salud percibida y nivel educativo en España: tendencias por comunidades autónomas y sexo 20012012. Gac Sanit. 2015;29(1):37-43. Disponible en: https://doi.org/10.1016/j.gaceta.2014.07.004

28. Torrents Roser RM, Ferreiro M, López $A$, Renedo $L$, Lleixà $M$, et al. Ansiedad en los cuidados: una mirada desde el modelo de Mishel. Índex Enferm (Granada) Enero-junio 2013;22(1-2):60-64. ISSN 1665-7063. Disponible en: http://dx.doi. org/10.4321/S1132-12962013000100013

29. Burbano López C, Sánchez LE. Traumatismo de la médula espinal e incertidumbre desde la teoría de Merle Mishel. Enfermería Universitaria. 2017;14(3):176-183. Disponible en: http://dx.doi.org/10.22201/ eneo.23958421e.2017.3.61241

30. Vera Romero OE, Vera Romero FM. Evaluación del nivel socioeconómico: presentación de una escala adaptada en una población de Lambayeque. Rev. Cuerpo Méd. Hospital Nacional Almanzor Aguinaga Asenjo HNAAA (Chiclayo, Perú). Agosto 2013;6(1):41-45. ISSN 2227-4731. Disponible en: https://dialnet.unirioja.es/ descarga/articulo/4262712.pdf

31. Sanabria Castellanos CM, Suárez Robles MA, Estrada Montoya JH. Relación entre determinantes socioeconómicos, cobertura en salud y caries dental en veinte países. Rev. Gerencia y Políticas de Salud. 2015;14(28):161-189. Disponible en: http://dx.doi.org/10.11144/Javeriana. rgyps18-28.rdsc

32. Paolino $M$, et al. Determinantes sociales del seguimiento y tratamiento de mujeres con PAP anormal en Avellaneda. Rev Argent Salud Pública (Provincia de Buenos Aires) Septiembre 2011;2(8):2127. Disponible en: http://rasp.msal.gov.ar/ rasp/articulos/volumen8/art-orig-determinantes-sociales.pdf. 